ARTICLE

\title{
Spatial regulation of thermomorphogenesis by HY5 and PIF4 in Arabidopsis
}

\author{
Sanghwa Lee (i) ${ }^{1}$, Wenli Wang (i) ${ }^{1} \&$ Enamul Huq (iD) ${ }^{1 凶}$
}

Plants respond to high ambient temperature by implementing a suite of morphological changes collectively termed thermomorphogenesis. Here we show that the above and below ground tissue-response to high ambient temperature are mediated by distinct transcription factors. While the central hub transcription factor, PHYTOCHROME INTERCTING FACTOR 4 (PIF4) regulates the above ground tissue response, the below ground root elongation is primarily regulated by ELONGATED HYPOCOTYL 5 (HY5). Plants respond to high temperature by largely expressing distinct sets of genes in a tissue-specific manner. HY5 promotes root thermomorphogenesis via directly controlling the expression of many genes including the auxin and BR pathway genes. Strikingly, the above and below ground thermomorphogenesis is impaired in spaQ. Because SPA1 directly phosphorylates PIF4 and HY5, SPAs might control the stability of PIF4 and HY5 to regulate thermomorphogenesis in both tissues. These data collectively suggest that plants employ distinct combination of SPA-PIF4HY5 module to regulate tissue-specific thermomorphogenesis.

\footnotetext{
${ }^{1}$ Department of Molecular Biosciences and The Institute for Cellular and Molecular Biology, The University of Texas at Austin, Austin, TX, USA.

凶email: huq@austin.utexas.edu
} 
igh ambient temperature due to global warming affects all terrestrial life forms including crop yield ${ }^{1}$. Being rooted in soil, sessile plants respond to high ambient temperature by changing their architecture in a developmental process termed thermomorphogenesis, which is characterized by elongated hypocotyl, petiole and root tissues, hyponastic growth, reduced stomatal density and early flowering ${ }^{2-4}$. These morphological changes allow plants to cope with and complete reproductive cycle under high ambient temperature.

Plants perceive high ambient temperature using diverse mechanisms. These include the red-light photoreceptor phytochrome $\mathrm{B}(\mathrm{phyB})^{5,6}$, an RNA thermoswitch within the $5^{\prime}$ untranslated region of the PHYTOCHROME INTERCTING FACTOR 7 (PIF7) 7 and a prion-like domain in EARLY FLOWERING 3 (ELF3) ${ }^{8}$. Downstream of the thermosensors, PIF4 acts as a central hub transcription factor controlling target genes especially auxin biosynthesis and signaling genes to promote hypocotyl and petiole elongation ${ }^{9-12}$. In addition, a number of factors including Cryptochrome 1 (Cry1), ELF3, TOC1, HMR, BBX18/23, TCP, FCA, SPAs, HECATE1/2 (HEC1/2), COP1DET1-HY5 module, and GI-RGA-PIF4 module have been shown to regulate thermomorphogenesis ${ }^{13-25}$. All of these factors impinge upon PIF4 either controlling the PIF4 abundance and/or activity to regulate thermomorphogenesis.

High ambient temperature also affects root system architecture $^{26,27}$, which is linked to crop yield ${ }^{28,29}$. In sunflower and cotton, elevated temperature increases lateral root length and number ${ }^{27}$. In Arabidopsis, elevated temperature has been shown to increase root elongation by regulating Brassinosteroid (BR) signaling ${ }^{30}$. In addition, several reports showed that auxin signaling is also involved in root elongation under normal and high ambient temperature conditions ${ }^{31-33}$. For example, Feraru et al. showed that PIN-LIKES 6 (PILS6) acts as a negative regulator of root growth, and high ambient temperature-mediated destabilization of PILS6 abundance increases the nuclear availability of auxin to promote root elongation ${ }^{32}$. In addition, a recent report also showed that the shoot and root thermomorphogenesis are coordinated, and a shoot derived HY5/PIF regulatory module controls auxin biosynthetic and signaling genes to regulate root thermomorphogenesis ${ }^{34}$.

Our understanding of thermomorphogenesis is mostly based on the above ground tissues (e.g., hypocotyl and petiole elongation, flowering time $)^{3}$. However, the mechanistic details of how the below-ground tissues of plants respond to high ambient temperature is still rudimentary. Here we show that ELONGATED HYPOCOTYL 5 (HY5) acts as a key transcription factor in root thremomorphogenesis. The expression and stability of PIF4 and HY5 are spatially regulated by high ambient temperature. In addition, SPA kinases are necessary to stabilize both PIF4 and HY5 to regulate thremomorphogenesis in a tissue-specific manner.

\section{Results}

Analyses of spatial thermomorphogenesis at seedlings stage. To examine spatial regulation of thermomorphogenesis, we grew wild type (WT) Arabidopsis seedlings at either $22^{\circ} \mathrm{C}$ or $28^{\circ} \mathrm{C}$ under constant light (CL) up to seven days and measured the root and hypocotyl lengths over time (Fig. 1a, b). Thermo-induced primary root elongation occurred gradually over time (Fig. 1b, c) and the primary root length ratio of $28 / 22$ remained similar (Fig. 1c, d). Primary root length was also elongated at $28^{\circ} \mathrm{C}$ under long day (LD) conditions similar to that in CL, while the primary root length was even shorter at $28^{\circ} \mathrm{C}$ compared to $22^{\circ} \mathrm{C}$ under short day (SD) conditions, consistent with the previous report ${ }^{30}$ (Supplementary Fig. 1). In contrast, thermo-induced hypocotyl elongation occurred over time, and the hypocotyl length ratio of 28/22 increased over time and saturated at around five days (Fig. 1e, f). To further examine whether the thermo-induced primary root elongation can be observed under natural soilgrown conditions, we grew wild-type Arabidopsis seedlings for three days on $\mathrm{MS}$ plate at $22^{\circ} \mathrm{C}$ and then transferred to soil for additional five days at either $22^{\circ} \mathrm{C}$ or $28^{\circ} \mathrm{C}$ under LD conditions and measured the root length (Supplementary Fig. 2a-c). Similar to the growth conditions on MS plate, soil-grown seedlings displayed elongated roots at high ambient temperature. The hypocotyl thickness was also measured using light microscopy under the continuous light conditions (Supplementary Fig. 3a). The data show that the hypocotyl diameter was increased $\sim 30 \%$ after 4 days of treatment at $28{ }^{\circ} \mathrm{C}$ (Supplementary Fig. 3b), indicating that high ambient temperature promotes the thickness as well as elongation of hypocotyl. Overall, high ambient temperature affects seedling architecture of both above ground and belowground tissues profoundly.

HY5 is necessary for root thermomorphogenesis. PIF4 has been described as a key component in the hypocotyl and petiole elongation under high ambient temperature 9 . However, the thermo-induced root elongation was not affected in pif4 background ${ }^{30}$. To identify the factor(s) necessary for root thermomorphogenesis, we analyzed the primary root length phenotype of several mutants and overexpression lines under high ambient temperature (Fig. 2a-c). As expected, phyB-9 did not respond to high ambient temperature, whereas $P H Y B$ overexpression line showed similar response to WT, suggesting that phyB regulates both above and below-ground tissues at high ambient temperature. We also analyzed the primary root length phenotype of pif4-2 and pifQ at high ambient temperature to examine if PIFs are involved in root thermomorphogenesis (Fig. 2a, b, Supplementary Fig. 4). The data show that both pif4-2 and pifQ responded to root thermomorphogenesis with similar primary root length ratio of $28 / 22$, indicating that PIFs do not contribute to primary root elongation at high ambient temperature Supplementary Fig. $4 \mathrm{a}-\mathrm{c}$ ). Because HY5 has been shown to regulate thermomorphogenesis as well as root hair elongation ${ }^{14,35}$, we examined root thermomorphogenesis phenotype of hy5-215 at high ambient temperature. The results show that the primary root length of hy5-215 mutant did not alter at high ambient temperature, indicating that HY5 is necessary for the primary root elongation under high ambient temperature. Similar to the MS-grown seedlings, hy5-215 mutant did not show root thermomorphogenesis, while pifQ mutant responded to high ambient temperature in soil-grown conditions (Supplementary Fig. 2). Furthermore, to elucidate the genetic relationship of PIFs and HY5 in root thermomorphogenesis, the primary root length was measured for hy5-215 pifQ at high ambient temperature. As expected, the primary root length of hy5-215 pifQ did not respond to high ambient temperature, whereas pifQ displayed similar root length to that of wild-type (Fig. 2a-c), suggesting that hy5-215 is epistatic to pifQ for root thermomorphogenesis.

When we examined the hypocotyl thickness phenotype under high ambient temperature. Both hy5-215 and pifQ did not show any difference, while the WT displayed an increase in the hypocotyl thickness in response to high ambient temperature (Supplementary Fig. 5a-c). PIF4 overexpression line displayed an increased thickness constitutively compared to WT, while HY5 overexpression in the hy5 background complemented the phenotype. Moreover, epistatic analyses showed that the hypocotyl thickness phenotype of hy5-215 pifQ is similar to that of pifQ and hy5-215 (Supplementary Fig. 5a-c), suggesting that both PIFs and HY5 are acting in the same genetic pathway to 
a

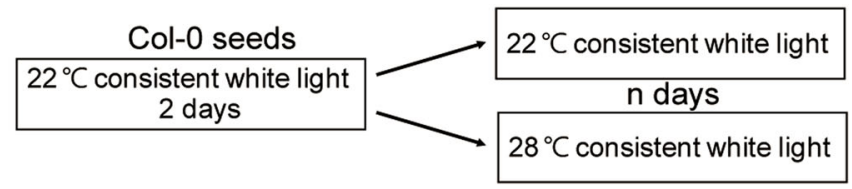

b

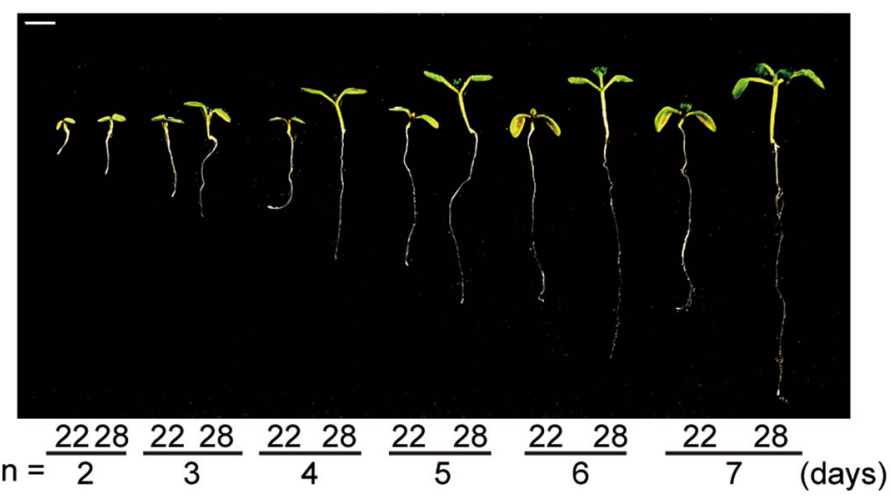

C

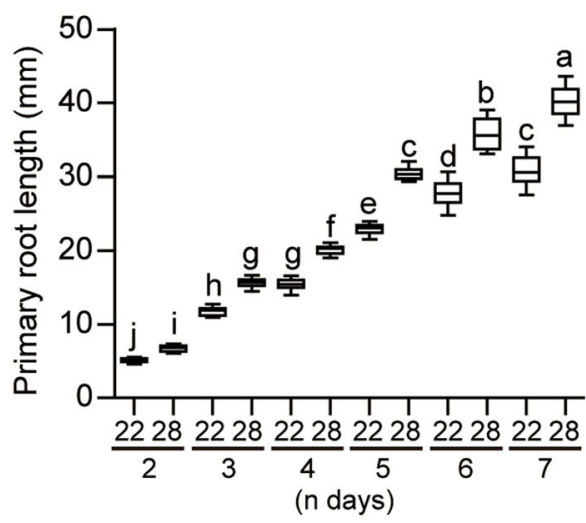

e

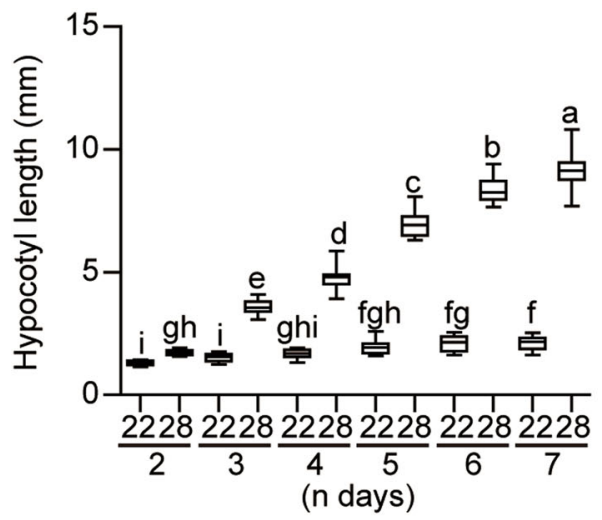

d

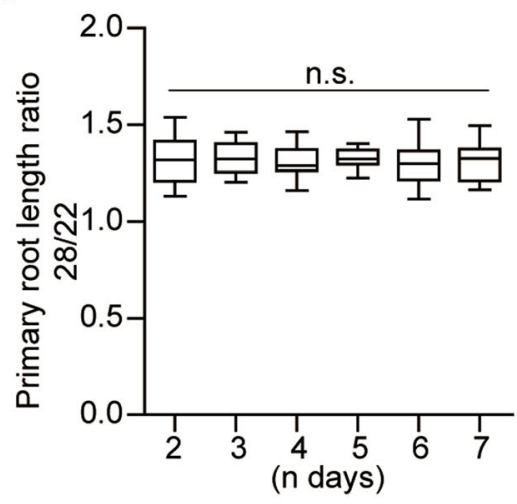

f

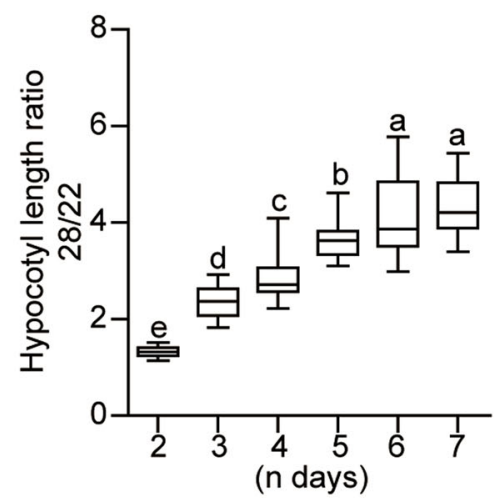

Fig. 1 Altered seedling architecture at high ambient temperature. a Simplified experimental condition used in this study for phenotypic analysis. b Photograph showing seedling phenotypes of Columbia- 0 grown at $22^{\circ} \mathrm{C}$ and $28{ }^{\circ} \mathrm{C}$ over time. Seedlings were grown for two days in continuous white light at $22{ }^{\circ} \mathrm{C}$ and then either kept at $22{ }^{\circ} \mathrm{C}$ or transferred to $28^{\circ} \mathrm{C}$ for additional $\mathrm{n}$ days before being photographed. The scale bar represents $2 \mathrm{~mm}$. c, e Bar graphs show the primary root (c) or hypocotyl (e) lengths for seedlings grown under conditions described in (a). d, $\mathbf{f}$ For the length ratio $28 / 22$, primary root or hypocotyl length at $28^{\circ} \mathrm{C}$ is divided by those values at $22^{\circ} \mathrm{C}$. The letters a-j indicate statistically significant differences between means of primary root or hypocotyl lengths $(n=15, P<0.05)$ based on one-way ANOVA analyses with Tukey's HSD test. n.s. stands for not significant. For the box and whisker plots in (c-f), the boxes represent from the 25 th to the 75 th percentile and the bars equal the median values. The source data underlying the root (c, d) and hypocotyl $(\mathbf{e}, \mathbf{f})$ measurements are provided in the Source data file.

regulate the hypocotyl thickness in response to high ambient temperature. Overall, these data suggest that HY5 is a key transcription factor for root thermomorphogenesis, while both PIFs and HY5 are acting in the hypocotyl tissue to regulate elongation as well as thickness in response to high ambient temperature.
HY5 controls global gene expression at elevated temperature. In thermomorphogenesis, an alteration in global gene expression has been reported mostly using whole seedlings $6,19,24$. To examine whether global gene expression is altered in a tissue-specific manner at high ambient temperature, WT and hy5-215 seedlings were grown at $22^{\circ} \mathrm{C}$ for 6 days under constant white light, and 
a

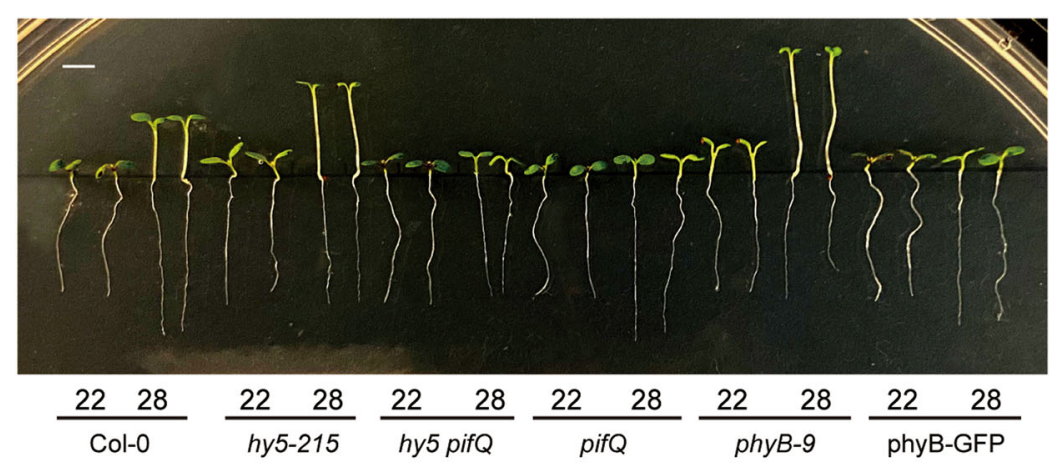

b

C
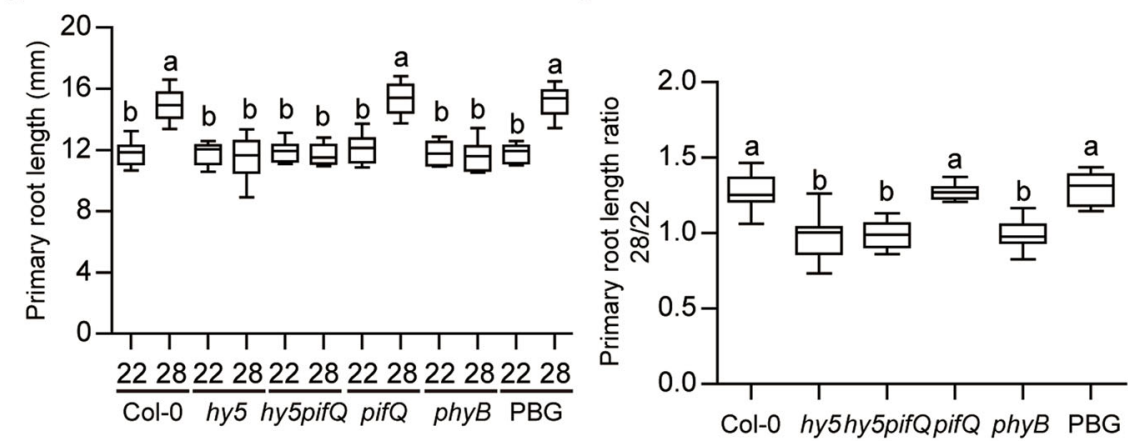

Fig. 2 HY5 is essential for root thermomorphogenesis. a Photograph showing seedling phenotypes of different mutants grown at $22^{\circ} \mathrm{C}$ and $28^{\circ} \mathrm{C}$. Seedlings were grown for 2 days in continuous white light at $22^{\circ} \mathrm{C}$ and then either kept at $22^{\circ} \mathrm{C}$ or transferred to $28^{\circ} \mathrm{C}$ for additional 4 days before being photographed. The scale bar represents $2 \mathrm{~mm}$. PBG stands for 35S:phyB-GFP. b, c Bar graphs show the primary root lengths for seedlings grown under conditions described in (a). For the length ratio $28 / 22$ (c), primary root length at $28^{\circ} \mathrm{C}$ is divided by that at $22^{\circ} \mathrm{C}$. The letters a, b indicate statistically significant differences between means of primary root lengths $(n=15, P<0.05)$ based on one-way ANOVA analyses with Tukey's HSD test. For the box and whisker plots in $(\mathbf{b}, \mathbf{c})$, the boxes represent from the 25 th to the 75 th percentile and the bars equal the median values. The source data underlying the root measurements $(\mathbf{b}, \mathbf{c})$ are provided in the Source data file.

then exposed to $28^{\circ} \mathrm{C}$ for additional $4 \mathrm{~h}$ before the shoot (hypocotyl and cotyledon) and root samples were collected. RNAseq analysis was performed using total RNA isolated from these samples. The results show that the shoot and root samples respond to high ambient temperature by differentially expressing genes (DEGs) that are largely distinct with partial overlap (Supplementary Fig. 6a, Supplementary data 1). Gene Ontology (GO) analysis of the shared 228 DEGs between the shoot and root showed an enrichment of temperature/heat/stress response genes including HSP90 (Supplementary Fig. 6b) ${ }^{33}$, suggesting that both organs respond to elevated temperature at least partially by controlling a common set of genes. However, the genes responding to high temperature in an organ-specific manner show an enrichment of different classes. For example, while genes involved in regulation of protein kinase activity, response to nitrogen compounds, mitotic cell cycle, response to abiotic stimulus show an enrichment in the shoot tissue (Supplementary Fig. 6c), the genes involved in CDP-diacylglycerol metabolic process, secondary metabolite biosynthetic process, cellular response to oxygen levels and oxidative stress are enriched in the root tissue (Supplementary Fig. 6d). These data suggest that the above ground and below-ground tissues respond to high ambient temperature differently.

When comparing the shoot samples from the WT Col-0 and hy5-215 in response to high ambient temperature, 1427 and 1083 DEGs were identified in the Col-0 and hy5-215, respectively, with 418 genes shared between the Col-0 and hy5-215 shoot samples (Fig. 3a). Importantly, $>70 \%$ of WT DEGs are HY5-dependent, supporting the previous reports that HY5 contributes to shoot thermomorphogenesis ${ }^{13,36}$. By contrast, 1129 and 846 genes were differentially expressed in the root samples of Col-0 and hy5-215, respectively, in response to high ambient temperature. In the root tissue, $>84 \%$ of Col-0 DEGs are HY5-dependent, suggesting a prominent role of HY5 in root thermomorphogenesis. Figure $3 \mathrm{~b}$ displays the heatmap analysis of the 1427 and 1129 DEGs from the WT Col-0 hypocotyl and root samples, respectively, in both WT and hy5-215, indicating that the gene expression is globally regulated by HY5 in both hypocotyl and root tissues. GO analysis of the HY5-dependent 1009 DEGs in the hypocotyl, and 956 DEGs in the root samples showed an enrichment of diverse processes that are largely distinct between the shoot and root tissues (Fig. 3c), suggesting that HY5 might regulate different pathways in a tissue-specific manner to respond to high ambient temperature.

We also compared our HY5-regulated genes to those of the HY5-bound genes that has been recently described ${ }^{37}$. These analyses show that $>50 \%$ of the HY5-regulated genes are also HY5-direct target genes (Supplementary Fig. 7a). When we compared at the organ-specific manner, $>51 \%$ of the HY5regulated genes in the shoot tissue are also HY5 direct target genes, while in the root sample, $>50 \%$ of the HY5-regulated genes are HY5 direct target genes (Supplementary Fig. 7a). Only 55 of the 939 potentially HY5-regulated and HY5-direct target genes are expressed in both organs (Supplementary Fig. 7a), suggesting that HY5 regulates a large proportion of genes in an organspecific manner to respond to high ambient temperature.

To confirm our RNAseq data, we selected 3 genes (IAA19, SAUR40, and SAUR77) involved in auxin signaling for independent verification by qPCR, as these genes have been linked to both root and hypocotyl elongation ${ }^{38-41}$. Consistent with our RNAseq 
a

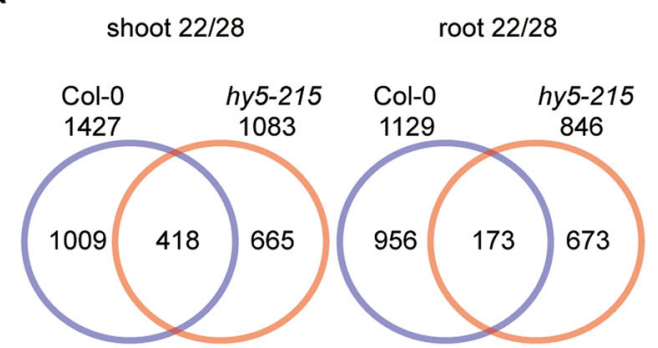

c
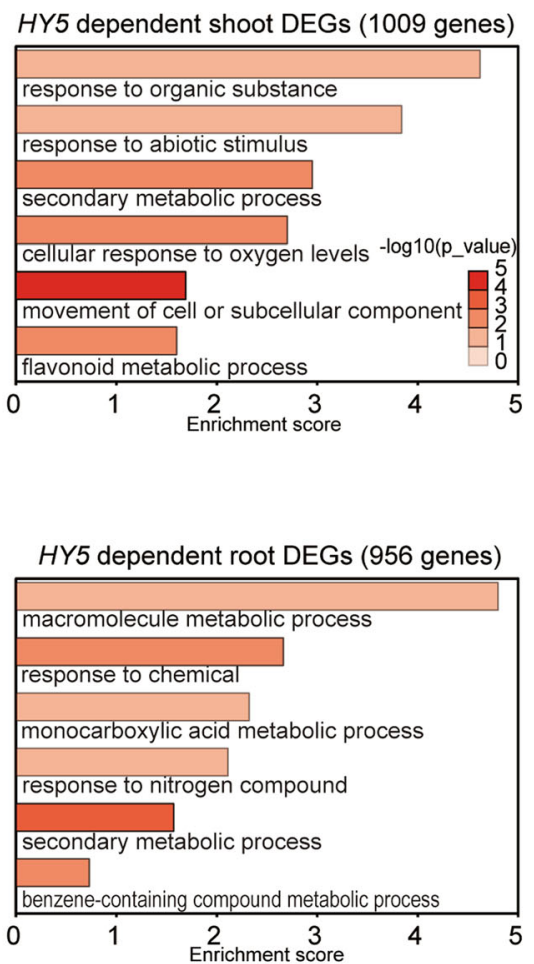

b

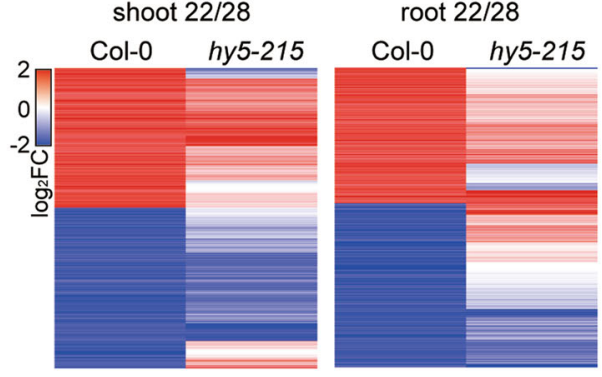

d

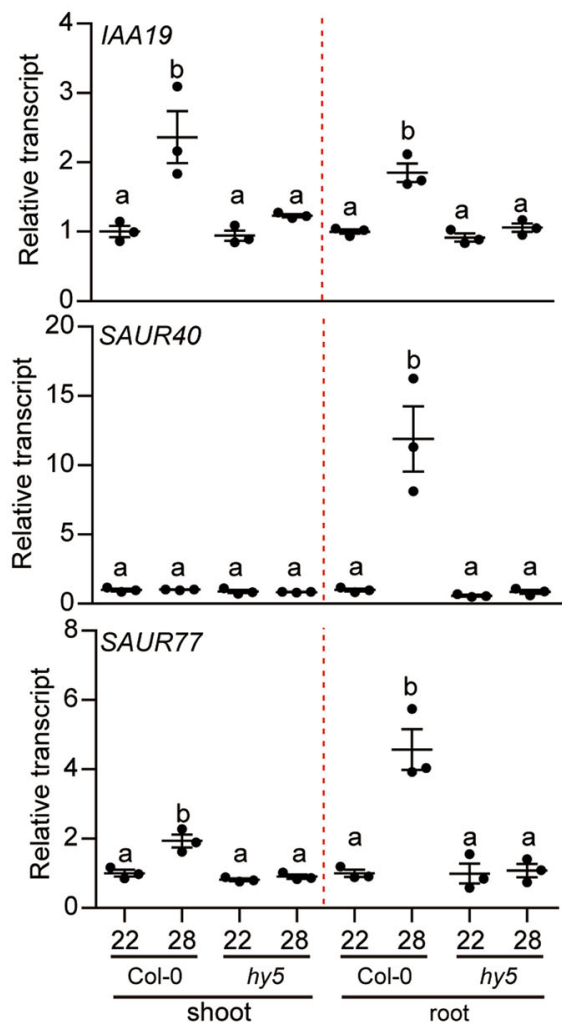

Fig. 3 HY5 controls global gene expression at elevated temperature in both shoot and root. a Venn diagrams show tissue-specific (shoot and root) Differentially Expressed Genes (DEGs) in wild-type (WT) vs hy5-215 mutant at high ambient temperature. Six-day-old white light-grown seedlings were transferred to $22^{\circ} \mathrm{C}$ or $28^{\circ} \mathrm{C}$ for additional $4 \mathrm{~h}$ and total RNA was extracted from three biological replicates for RNA-seq analyses. b Heatmap analysis shows 1427 and 1129 DEGs between wild-type and hy5-215 mutant shoot and root, respectively. c Gene Ontology (GO) analysis of HY5-dependent shoot and root DEGs. DEGs were identified with $\geq 2$-fold and FDR $\leq 0.05$. d RT-qPCR analysis using IAA19, SAUR40, and SAUR77. RT-qPCR samples were from Col-0 whole seedlings, shoot or root grown for 6 days at $22^{\circ} \mathrm{C}$ and then either kept at $22^{\circ} \mathrm{C}$ or transferred to $28^{\circ} \mathrm{C}$ for $4 \mathrm{~h}$. Three biological repeats were performed. Error bars indicate SD $(n=3)$. Relative gene expression levels were normalized using the expression level of ACT7 and the values of those genes in wild-type. The letters a, b indicate statistically significant differences $(n=3, P<0.05)$ based on one-way ANOVA analyses with Tukey's HSD test.

data (Supplementary Fig. 7b), the transcript levels of these genes were highly repressed in hy5-215 in both shoot and root samples (Fig. 3d). Strikingly, SAUR40 was highly expressed only in the WT root sample, indicating a root-specific HY5 regulation of SAUR40 at high ambient temperature (Fig. 3d, middle). These data are consistent with the previous report showing the SAUR40-EYFP expression in the root tip ${ }^{38}$. Since the auxin signaling genes (IAA19, SAUR40, SAUR77, and PILS6) are potentially direct targets of HY5 as the ChIPseq assays showed an enrichment of HY5 binding to the promoter regions of these genes (Supplementary Fig. 7c) ${ }^{37}$, we performed Chromatin Immunoprecipitation (ChIP) assays using 35S:HY5-GFP/hy5215 whole seedlings (Supplementary Fig. 7d). The result shows that the HY5 binding to these promoters was enriched at high ambient temperature, indicating that HY5 directly binds to the promoter regions of IAA19, SAUR40, and SAUR77. Taken together, these data suggest that HY5 promotes root thermomorphogenesis by regulating gene expression globally in root tissue.

HY5 promotes BR-mediated root elongation at high temperature. A recent study showed that the primary root elongation at high ambient temperature is also mediated by the brassinosteroid (BR) signaling pathway ${ }^{30}$. Therefore, we examined whether the thermo-insensitive primary root phenotype of hy5-215 mutant is caused in part by an alteration in the expression of BR metabolic genes. Although these genes were not identified in our RNAseq analyses, qRT-PCR assays show that the transcript 
level of the BR biosynthetic gene $C P D$ was upregulated, while that of BR catabolic genes BAS1 and SOB7 was downregulated in the WT root tissue at high ambient temperature as previously reported ${ }^{30}$. However, the transcript level of these genes did not alter in the hy5-215 mutant in response to high ambient temperature (Supplementary Fig. 8a). In contrast, the expression of these genes was restored in the 35S:HY5-GFP/hy5-215 complemented line, supporting that HY5 is essential for triggering thermo-induced regulation of the BR metabolic pathway (Supplementary Fig. 8a). Furthermore, because HY5 is a bZIP protein transcription factor that binds to several DNA motifs such as Gbox (CACGTG), CG hybrid (GACGTG), CA hybrid (GACGTA) or ACGTG motifs $37,42,43$, we searched the shared sequence of these motifs (ACGT motif) within the CPD, BAS1, and SOB7 promoter regions. Among the three genes, the promoter regions of $B A S 1$ and $S O B 7$ contain multiple HY5-binding sites within the $2 \mathrm{~kb}$ promoter regions ( 3 in $B A S 1$ and 8 in SOB7) (Supplementary Fig. 8b). Therefore, we performed ChIP assays using 35S:HY5GFP overexpressed in the hy5-215 mutant background to examine whether HY5 directly binds to the promotor region of these genes. As expected, an enrichment of the HY5 binding was observed in the promoter region containing the ACGT sites (P2 in BAS1 and $\mathrm{P} 3$ in SOB7) in response to high ambient temperature compared to the control regions (P1 in BAS1 and $A C T 7$ ), indicating that HY5 directly binds to the BR metabolic genes (BAS1 and SOB7) and controls their expression. Overall, these data suggest that HY5 might also regulate root thermomorphogenesis by controlling the BR signaling pathway.

Phosphorylation of HY5 promotes root thermomorphogenesis. To examine whether HY5 protein level is regulated by high ambient temperature, we used the HY5-GFP overexpression line driven by the constitutive cauliflower mosaic virus (CaMV) $35 \mathrm{~S}$ promoter in the hy5-215 mutant background. While the HY5GFP transcript level is not altered at high ambient temperature (Supplementary Fig. 9), immunoblot analysis showed that HY5GFP accumulated at high ambient temperature in both shoot and root tissues of WT seedlings (Supplementary Fig. 10). These data suggest that the altered HY5 protein level might be due to posttranslational regulation.

Recently, we have shown that SPA1 can directly phosphorylate HY5 at serine 36 residue and regulate its abundance and activity, consistent with the previous reports ${ }^{44,45}$. These reports showed that the unphosphorylated HY5 is more active but also more unstable, because it is a preferred substrate for COP1-mediated degradation. Since HY5 abundance is post-translationally regulated, we hypothesized that the phosphorylation of HY5 might regulate HY5 stability and activity. Therefore, we analyzed two additional forms of HY5 fused with GFP; phospho-null HY5S36A, and phospho-mimic HY5-S36D overexpression lines driven by the CaMV35S promoter in the hy5-215 mutant background ${ }^{44}$. Analyses of the root thermomorphogenesis phenotype showed that all three transgenic plants display similar primary root length at $22^{\circ} \mathrm{C}$. However, the phospho-null HY5S36A form did not respond to high ambient temperature, whereas the phospho-mimic HY5-S36D form showed longer primary root length at high ambient temperature similar to the wild-type HY5 overexpression line (Fig. 4a-c). These data suggest that phosphorylation of HY5 is essential for root thermomorphogenesis. To check whether HY5 protein level is altered at high ambient temperature in a tissue-specific manner, western blot was performed (Supplementary Fig. 10). While three different HY5 forms show similar level at normal condition, WT HY5-GFP and HY5-S36D were more abundant, whereas HY5-S36A showed decreased level at high ambient temperature in both shoot and root tissues. These data show that phosphorylation of HY5 is essential for thermo-induced stabilization. To further analyze whether HY5 protein level is regulated in a tissue-specific manner, fluorescent microscopy was used to detect GFP signal in the root tissues (Fig. 4d, e). Similar to the immunoblot (Supplementary Fig. 10), wild-type HY5-GFP signal was accumulated in the entire root tissue at high ambient temperature (Fig. 4d, e). However, HY5-S36A showed reduced GFP signal, while HY5-S36D showed accumulated GFP signal similar to the wild-type HY5-GFP at high ambient temperature (Fig. 4d, e). Although GFP fusion may affect the expression and stability of a fusion protein, overall these data suggest that the phosphorylation of HY5 is necessary to stabilize HY5 in the root to promote primary root elongation at high ambient temperature.

SPAs are necessary for root thermomorphogenesis. Recently, we showed that SPA proteins are necessary for the thermomorphogenesis phenotype of the shoot ${ }^{24}$. To examine whether the SPAs are also involved in regulating root thermomorphogenesis, we tested high ambient temperature-mediated root elongation phenotype using spa higher order mutants (spa123 and spa1234, spaQ) (Fig. 5a, b). Results show that the thermo-induced root elongation was attenuated in the spa123 and absent in $s p a Q$ mutant compared to WT. The primary root length was even shorter for $\operatorname{spaQ}$ at $28^{\circ} \mathrm{C}$ vs $22^{\circ} \mathrm{C}$, suggesting that all four SPA genes are necessary for root elongation.

Lee et al. also showed that SPA1 directly phosphorylates and stabilizes PIF4 to promote hypocotyl and petiole elongation ${ }^{24}$. To test whether the kinase activity of SPA1 is necessary for root thermomorphogenesis, we grew previously described transgenic plants expressing the WT SPA1 (35S:LUC-SPA1/spaQ) or mutant SPA1 (35S:LUC-mSPA1/spaQ) affecting its kinase activity in the spaQ background ${ }^{46}$. Examination of the primary root length phenotype showed that the WT SPA1 can largely rescue the root elongation phenotype of spaQ. However, the mutant SPA1 affecting its kinase activity failed to rescue the root elongation phenotype of $s p a Q$ at high ambient temperature (Fig. 5a-c), suggesting that the kinase activity of SPA1 is necessary for root thermomorphogenesis.

To test more directly whether the kinase activity of SPA1 is regulated by temperature, we performed in vitro kinase assays using WT HY5 as a substrate at normal $\left(22^{\circ} \mathrm{C}\right)$ and high ambient temperature $\left(28^{\circ} \mathrm{C}\right)$. Strikingly, the results show that the kinase activity of SPA1 is stimulated $>2$-fold at $28^{\circ} \mathrm{C}$ (Supplementary Fig. 11). Given that HY5 is stabilized by SPA1-mediated phosphorylation of serine 36 of HY5 (Fig. 4d, e; Supplementary Fig. 10) ${ }^{44}$, these data suggest that SPA1 might phosphorylate HY5 more at high ambient temperature to stabilize it. This is also consistent with the SPA1-mediated phosphorylation and stabilization of PIF $4^{24}$.

We also examined the hypocotyl thickness phenotypes of the above lines in response to high ambient temperature (Supplementary Fig. 12). Results show that the thickness of spaQ hypocotyl at $22^{\circ} \mathrm{C}$ was higher than that of WT at $28^{\circ} \mathrm{C}$. Moreover, spaQ hypocotyl became much thicker at $28^{\circ} \mathrm{C}$ compared to $22^{\circ} \mathrm{C}$ possibly due to reduced elongation. To test whether the kinase activity is necessary for the hypocotyl thickness phenotype, we also analyzed the transgenic plants expressing the WT SPA1 (35S:LUC-SPA1/spaQ) or mutant SPA1 (35S:LUC-mSPA1/spaQ) affecting its kinase activity in the spaQ background as described above. The data show that the WT SPA1 largely rescued the hypocotyl thickness phenotype of the spaQ. By contrast, the mutant SPA1 slightly reduced the hypocotyl thickness of the spaQ mutant in response to high ambient temperature. These data suggest that the kinase activity of SPA1 is 
a

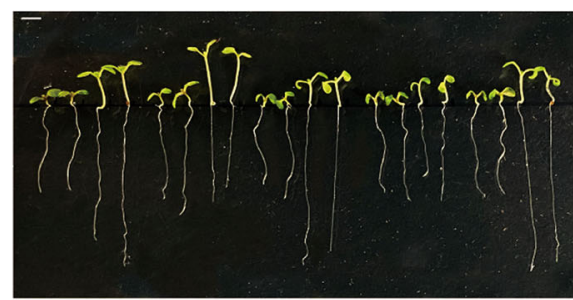

$\frac{22 \quad 28}{\text { Col-0 } \frac{2228}{-\quad} \frac{2228}{H Y 5(\mathrm{WT})} \frac{2228}{\mathrm{HY} 5(\mathrm{~A})} \frac{2228}{\mathrm{HY} 5(\mathrm{D})}}$

b

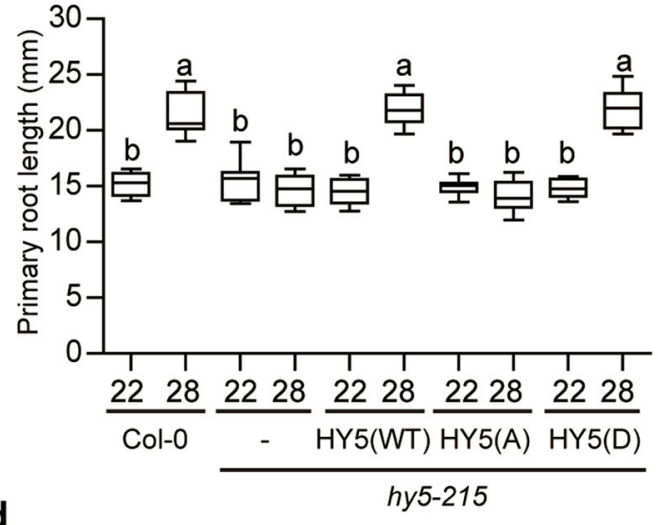

C
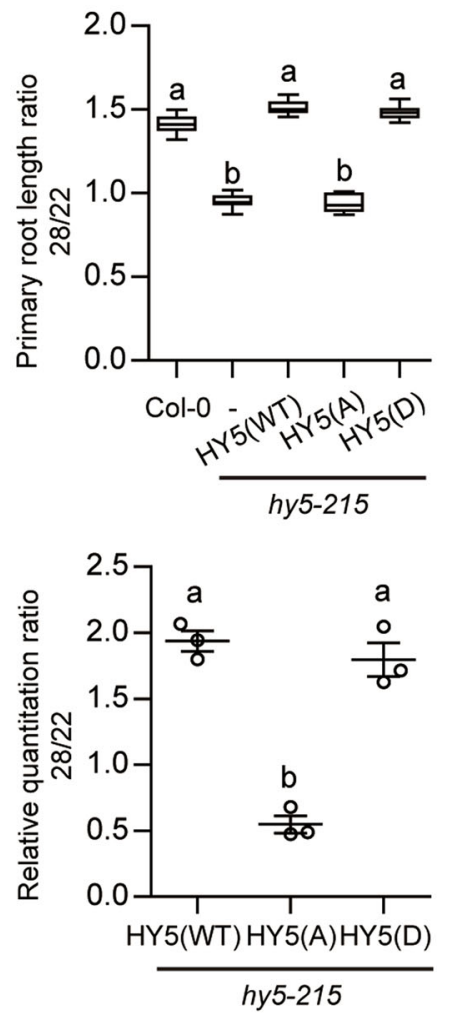

Fig. 4 Phosphorylation of HY5 is necessary for stabilization and root thermomorphogenesis. a Photograph showing seedling phenotypes of three different types of $\mathrm{HY} 5$ overexpression lines grown in $22^{\circ} \mathrm{C}$ and $28^{\circ} \mathrm{C}$. Seedlings were grown for two days in continuous white light at $22^{\circ} \mathrm{C}$ and then either kept at $22^{\circ} \mathrm{C}$ or transferred to $28^{\circ} \mathrm{C}$ for additional 4 days before being photographed. The scale bar represents $2 \mathrm{~mm}$. b, c Bar graphs show the primary root lengths (b) or the root length ratio (c) for the seedlings grown under conditions described in (c). For the length ratio $28 / 22$, the primary root length at $28^{\circ} \mathrm{C}$ is divided by that at $22^{\circ} \mathrm{C}$. The letters a-c indicate statistically significant differences between means of primary root lengths $(n=12, P<0.05)$ based on one-way ANOVA analyses with Tukey's HSD test. For the box and whisker plots in $\mathbf{b}$, $\mathbf{c}$, the boxes represent from the 25th to the 75th percentile and the bars equal the median values. The source data underlying the root measurements $(\mathbf{b}, \mathbf{c})$ are provided in the Source data file. $\mathbf{d}$ Fluorescence images of roots using three types of HY5-GFP overexpression lines with the same exposure time $(t=300 \mathrm{~ms})$. The scale bar represents $200 \mu \mathrm{m}$. e Bar graph shows the relative amount of HY5-GFP signal at $28^{\circ} \mathrm{C}$ divided by that at $22^{\circ} \mathrm{C}$ with three repeats. Error bars indicate SD $(n=3)$. The letters a, b indicate statistically significant differences between means of primary root lengths $(n=3, P<0.05)$ based on one-way ANOVA analyses with Tukey's HSD test.

also necessary for regulating the hypocotyl thickness in response to high ambient temperature.

PIF4 and HY5 are spatially regulated. Because PIF4 and HY5 appear to regulate thermomorphogenesis largely in a tissuespecific manner, we tested the expression of these genes using whole seedlings, shoot and root tissues grown at $22^{\circ} \mathrm{C}$ and then transferred to $28^{\circ} \mathrm{C}$ for $4 \mathrm{~h}$. qPCR analyses showed that the expression of PIF4 is upregulated only in the shoot tissues with similar level in the root (Fig. 6a). Strikingly, the expression of HY5 showed specific upregulation only in the root tissue (Fig. 6b), with similar expression in the shoot and total seedlings in response to high ambient temperature. Although the growth conditions are slightly different, these expression data are largely consistent with the expression of PIF4 and HY5 in publicly available datasets (Supplementary Fig. 13).

To test whether the expression pattern of these genes reflects the protein level in these tissues, we also performed immunoblot analyses of PIF4 and HY5 using shoot and root tissue samples grown under the same conditions. Results show that PIF4 is more abundant in the shoot, while the HY5 level is upregulated specifically in the root in response to high ambient temperature 


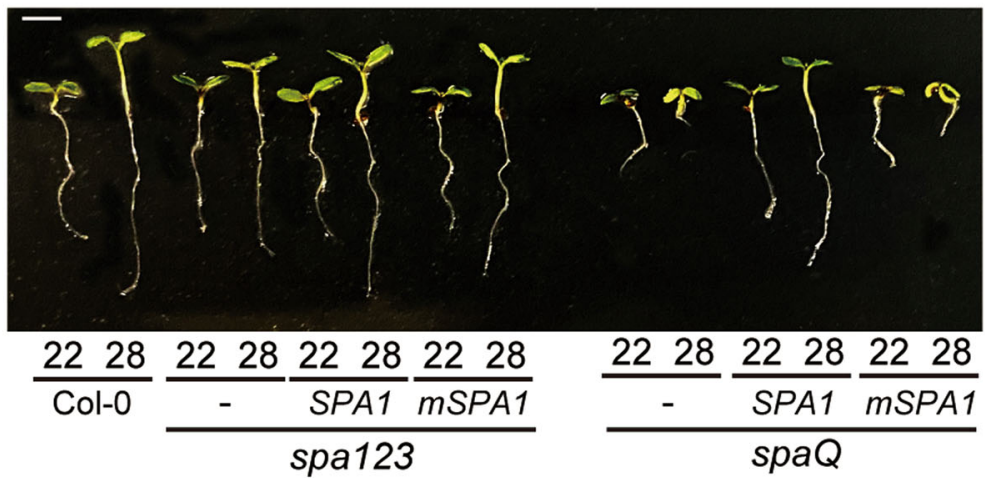

b

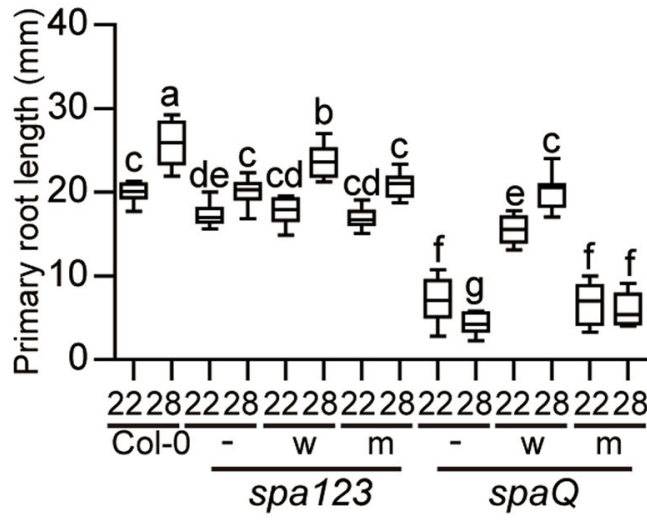

C

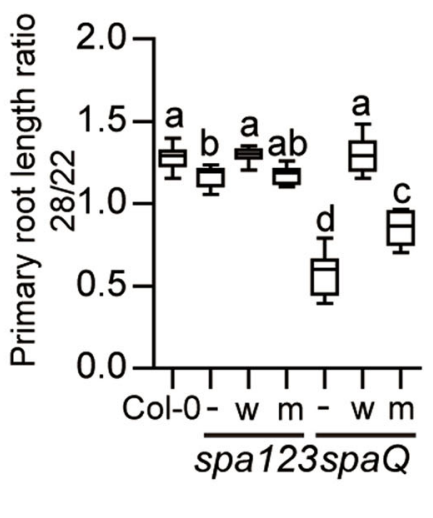

Fig. 5 SPAs are necessary for root thermomorphogenesis. a Photograph shows the seedling phenotypes of Col-0, spa123, 35S:LUC-SPA1/spa123, and 35S: LUC-mSPA1/spa123, spaQ, 35S:LUC-SPA1/spaQ, and 35S: LUC-mSPA1/spaQ at $22^{\circ} \mathrm{C}$ and $28^{\circ} \mathrm{C}$. Three spa background plants are indicated with-for spa123 or spaQ, SPA1 for 35S:LUC-SPA1 overexpression line, and mSPA1 for 35S:LUC-mSPA1 overexpression line, respectively. Seedlings were grown for two days in continuous white light at $22^{\circ} \mathrm{C}$ and then either kept at $22^{\circ} \mathrm{C}$ or transferred to $28^{\circ} \mathrm{C}$ for additional 5 days before being photographed. The scale bar represents $2 \mathrm{~mm}$. b, c Bar graph shows the primary root lengths (b) and the root length ratio (c) for seedlings grown under conditions described in (a). W, wild-type SPA1 and m, mSPA1, respectively. For the length ratio $28 / 22$, the primary root length at $28^{\circ} \mathrm{C}$ was divided by that at $22{ }^{\circ} \mathrm{C}$. The letters a-f indicate statistically significant differences between means of primary root lengths $(n=15, P<0.05)$ based on one-way ANOVA analyses with Tukey's HSD test. For the box and whisker plots in $\mathbf{b}, \mathbf{c}$, the boxes represent from the 25 th to the 75 th percentile and the bars equal the median values. The source data underlying the root measurements $(\mathbf{b}, \mathbf{c})$ are provided in the Source data file.

(Fig. 6c). In fact, HY5 level is slightly reduced in the shoot tissue in response to high ambient temperature (Supplementary Fig. 14), while PIF4 remains unchanged in the root tissue. To examine whether the phenotypic difference of root thermomorphogenesis under LD and SD reflects the protein levels, immunoblot of HY5 was performed using samples grown under LD and SD conditions (Supplementary Fig. 15). The results show that while the basal level of HY5 in the root was higher under LD compared to SD conditions, HY5 was more stabilized by high ambient temperature under LD compared to SD conditions. While the lack of stabilization of $\mathrm{HY} 5$ at $28^{\circ} \mathrm{C}$ compared to $22^{\circ} \mathrm{C}$ under SD vs LD may not solely explain the differences in root thermomorphogenesis phenotype under these conditions, these data suggest that proper level of HY5 in the root might be essential for triggering root thermomorphogenesis. Overall, these tissue-specific gene expression and protein abundance of PIF4 and HY5 might explain why plants employ these two transcription factors to regulate thermomorphogenesis in a tissue-specific manner.

\section{Discussion}

Global warming caused by climate change has a profound impact on crop productivity ${ }^{1,47}$. However, our understanding of the impact of high ambient temperature is mostly based on the above ground tissue responses, 2,3 . Our comprehensive phenotypic, genetic, biochemical and genomic analyses show that plants respond to high ambient temperature in a tissue-specific manner and employ distinct transcription factors to regulate spatial thermomorphogenesis.

Root system architecture plays a crucial role in crop productivity $28,29,48$ and high ambient temperature affects both primary and lateral root growth ${ }^{26,27,30}$. The phenotypic analyses show that seedlings display a general growth promotion including elongation of hypocotyls, petioles and roots, and thickening of hypocotyls in response to high ambient temperature. Moreover, day-length profoundly affects growth responses with stronger impact on root elongation under SD conditions. This is in sharp contrast where hypocotyl elongates more under SD compared to $\mathrm{LD}$ or constant light conditions. In addition, global gene expression analyses show that root and shoot respond to high ambient temperature largely differently with a small set of overlapping genes. These data are consistent with the previous reports that plants respond to high ambient temperature in a tissue-specific manner ${ }^{30,34}$.

Previously, it was shown that PIF4 acts as a main hub transcription factor regulating plant responses to high ambient temperature ${ }^{9}$. PIF4 plays a minor, if any role, in root responses to high ambient temperature ${ }^{30}$. Our genetic analyses suggest that while PIF4 acts a hub transcription factor regulating the above ground tissue-responses to high ambient temperature, HY5 acts as a key transcription factor regulating root responses to high ambient temperature. A recent report concluded that PIF4 is 
a

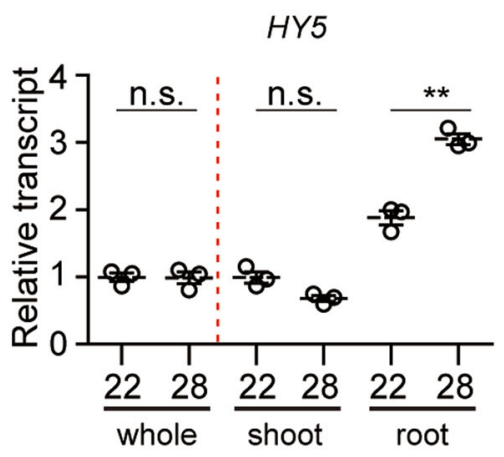

b

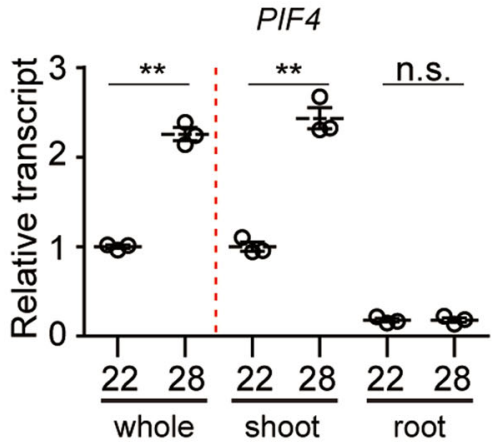

C

Col-0

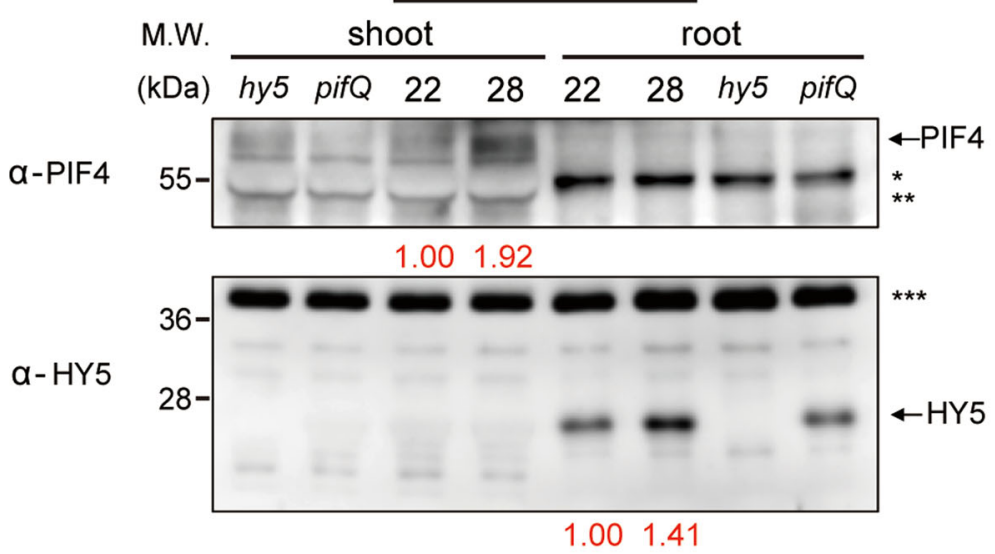

CBB

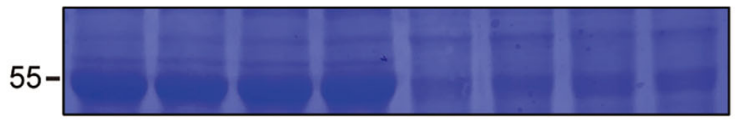

d

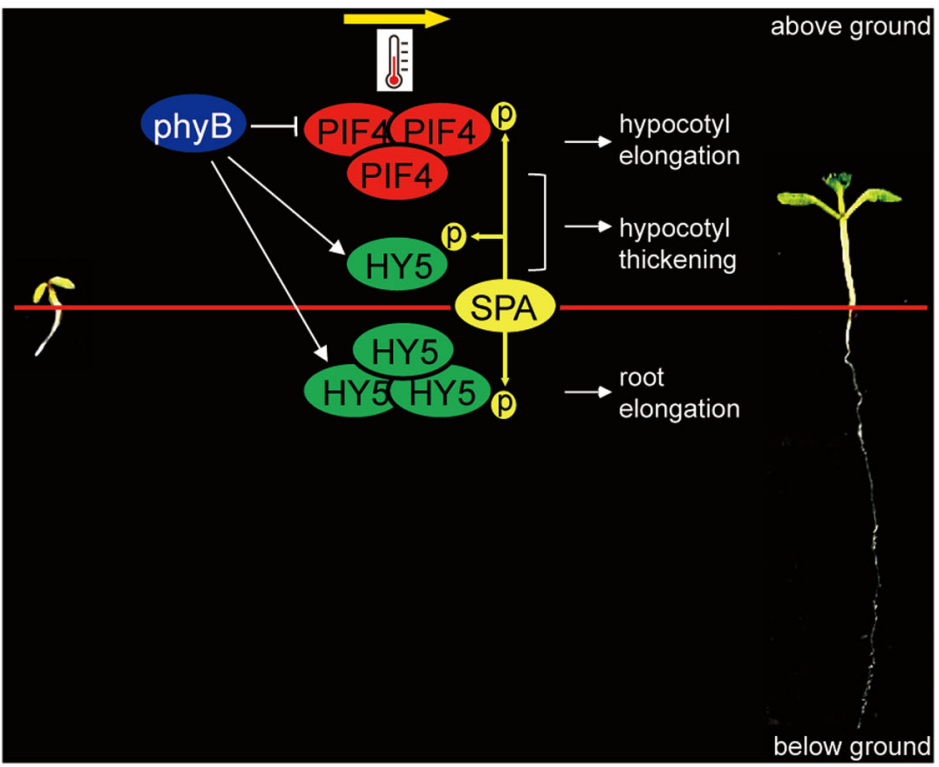

involved in root thermomorphogenesis based on PIF4 overexpression line ${ }^{34}$. However, our genetic analyses data using pif4-2 and pifQ mutants are consistent with a previous conclusion that PIF4 does not play a role in root thermomorphogenesis ${ }^{30}$. These data are also consistent with the tissue-specific expression and protein abundance of PIF4 and HY5 in shoot and root, respectively.
The genomic analyses suggest that HY5 directly/indirectly regulates a large number of genes in both shoot and root tissues consistent with its role as previously shown $37,42,43,49$. However, the shoot and root-specific transcriptomic changes are largely distinct with a small overlapping set, highlighting a distinct role of HY5 in regulating the above and below-ground tissue responses to high ambient temperature. A more in-depth gene 
Fig. 6 The expression and stability of PIF4 and HY5 are spatially regulated. a, b RT-qPCR analysis of HY5 and PIF4. RT-qPCR samples were from Col-O whole seedlings, shoot or root grown for 5 days at $22^{\circ} \mathrm{C}$ and then either kept at $22^{\circ} \mathrm{C}$ or transferred to $28^{\circ} \mathrm{C}$ for 4 h. Three biological repeats were performed. Relative gene expression levels were normalized using the expression levels of ACT7 as well as the expression levels of shoot. Asterisks indicate statistically significant difference using two-sided Student's $t$-test; ${ }^{\star \star} p<0.01$. P values for HY5 root 22 vs 28: 0.0009, PIF4 whole seedlings: 0.0005 , PIF4 shoot: 0.0004 , respectively. Error bars indicate SD $(n=3)$. c Western blots show the level of native PIF4 and HY5 in the shoot and root samples separated from whole seedlings grown for 5 days at $22^{\circ} \mathrm{C}$ and then either kept at $22^{\circ} \mathrm{C}$ or transferred to $28^{\circ} \mathrm{C}$ for $4 \mathrm{~h}$. hy5-215 and pifQ were used as negative controls. ${ }^{*}$ and ${ }^{\star \star}$ indicate root and shoot specific cross-reacting bands. ${ }^{\star * \star}$ indicate cross-reacting band in HY 5 blot. Red numbers indicate the relative values for PIF4 or HY5 band divided by the cross-reacting band. Two independent experiments were repeated with similar results. $\mathbf{d}$ Simplified scheme showing spatial regulation of thermomorphogenesis by distinct transcription factor-kinase module. PIF4 and HY5 are main hub transcription factors in the shoot and root thermomorphogenesis, respectively, while PIF4 and HY5 both contribute to hypocotyl thickness. The abundance and/or activity of PIF4 and HY5 are regulated by SPA-mediated phosphorylation. As a thermosensor, phyB directly inhibits PIF4 and indirectly stabilizes HY5 to coordinately regulate shoot and root thermomorphogenesis.

expressing analyses using both RNAseq and qRT-PCR showed that HY5 directly regulated the expression of both auxin and BR pathway genes to regulate root thermomorphogenesis consistent with the previous reports ${ }^{30,34}$. HY5 protein has been shown to transport from shoot to root ${ }^{50}$. Using shoot-specific expression of HY5 ( $p C A B 3: D O F-H Y 5)$, Gaillochet et al. also showed that the shoot derived HY5 partially rescues the root thermomorphogenesis phenotype of hy $5^{34}$. However, our data show that HY 5 gene expression and protein stability are both stimulated by high ambient temperature in a root-specific manner, suggesting that the root-derived HY5 might play a large role in regulating root thermomorphogenesis.

Recently, we have shown that SPAs play a crucial role in regulating thermomorphogenesis by controlling the phyB-PIF4 module ${ }^{24}$. Moreover, SPA1 directly phosphorylates and stabilizes PIF4 to regulate thermomorphogenesis. In addition, SPAs directly phosphorylate and stabilize $\mathrm{HY}^{44}$. Unphosphorylated HY5 is more active, but a preferred substrate for COP1-mediated degradation as previously shown ${ }^{44,51}$. The phenotypic data show that SPAs regulate both shoot and root thermomorphogenesis, and the kinase activity of SPA1 is not only necessary for the biological function of SPA1, but also stimulated by high ambient temperature. Thus, SPAs might directly phosphorylate both PIF4 and HY5 to regulate the above- and below-ground tissue responses to high ambient temperature (Fig. 6d).

While HY5 regulates root thermomorphogenesis, our data show that its role is not only restricted to the root tissue. In fact, both HY5 and PIFs are involved in regulating hypocotyl thickness in response to high ambient temperature in an interdependent manner. Moreover, SPA kinase activity is also involved in regulating hypocotyl thickness. While PIFs and HY5 have been shown to regulate light and temperature responses in an antagonistic manner ${ }^{36}$, PIF1/PIF3 and HY5/HYH have been shown to interact with each other and coordinately regulate reactive oxygen species signaling in Arabidopsis ${ }^{52}$. Thus, PIFs-HY5-SPA module might regulate hypocotyl thickness in the above ground tissue. Further studies are necessary to understand how this regulatory module controls hypocotyl thickness in response to high ambient temperature.

In summary, the data presented here suggest that plants employ distinct transcription factors for regulating thermomorphogenesis in a tissue-specific manner. While PIF4-SPA module plays a pivotal role in hypocotyl elongation ${ }^{24}$, HY5-SPA module plays a crucial role in regulating root thermomorphogenesis (Fig. 6d). In addition, PIFs-HY5-SPA module regulates hypocotyl thickness in response to high ambient temperature. Moreover, a number of factors including, Cryptochrome 1 (Cry1), ELF3, TOC1, HMR, BBX18/23, TCP, FCA, COP1DET1-HY5 module, and GI-RGA-PIF4 module converges on PIF4 to control its expression and/or abundance to regulate shoot thermomorphogenesis ${ }^{13-24}$. Further studies are necessary to understand if any of these factors or others also regulate root thermomorphogenesis in concert with HY5.

\section{Methods}

Plant materials, growth conditions, and phenotypic analyses. All the seeds used in this study are from Col-0 ecotype of Arabidopsis thaliana. Seeds were surfacesterilized and plated on Murashige and Skoog (MS) media without any sucrose. Seeds were stratified for 3 days at $4{ }^{\circ} \mathrm{C}$, and then were placed in $22^{\circ} \mathrm{C}$ for 2 days and transferred to $22^{\circ} \mathrm{C}$ or $28^{\circ} \mathrm{C}$ for additional 4 days or as indicated in figure legends. For soil-grown root elongation experiments, seeds were germinated on MS plates for 3 days and then the seedlings were transferred to soil and grown for additional 5 days at either $22^{\circ} \mathrm{C}$ or $28^{\circ} \mathrm{C}$ under LD conditions. White light intensity was 30 $\mu \mathrm{mol} \mathrm{m} \mathrm{m}^{-2} \mathrm{~s}^{-1}$. Hypocotyl length, hypocotyl thickness, and primary root length with 10-15 seedlings were measured using ImageJ software and analyzed using one-way ANOVA with Tukey's HSD test.

Protein extraction and western blot analyses. Total protein extracts were made from 50 seedlings for each shoot or root sample using $50 \mu \mathrm{L}$ urea extraction buffer

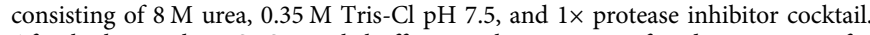
After boiling with $6 \times$ SDS sample buffer, samples were centrifuged at $20,200 \times g$ for $15 \mathrm{~min}$ and loaded into SDS-PAGE gels. Separated proteins were transferred onto PVDF membrane (Millipore) and then immunoblotted using anti-GFP (Abcam, ab290, dilution 1:5000), anti-PIF4 (Agrisera, AS16 3955, dilution 1:2000), or antiHY5 (Abiocode, R1245-2, dilution 1:3000) antibodies. For the secondary antibodies, anti-mouse (Abcam, ab131368, dilution 1:5000), anti-rabbit (KPL, 95059 086, dilution 1:50,000), or anti-goat (Agrisera, AS09 605, dilution 1:5000) were used. Coomassie blue staining (CBB), anti-Tubulin (Sigma-Aldrich, T5168, dilution 1:5000), or anti-RPT5 (Enzo Life Sciences, BML-PW8770-0025 dilution 1:5000), was used for loading control. SuperSignal West Atto Chemiluminescent substrate (ThermoFisher Scientific, A38556) was used for visualizing secondary HRP bound antibodies.

RNA extraction, cDNA synthesis, and qRT-PCR. Whole seedlings, shoot and root tissues from 6-day-old white light-grown seedlings were used with three independent biological replicates $(n=3)$ for RNA extraction. Seedlings were grown at $22^{\circ} \mathrm{C}$ under continuous white light for 6 days and then were either kept at $22^{\circ} \mathrm{C}$ or transferred to $28^{\circ} \mathrm{C}$ for additional $4 \mathrm{~h}$ under continuous white light before samples were harvested. Plant RNA purification kit from Sigma-Aldrich were used for total RNA extraction following the manufacturer's protocols. For cDNA synthesis, $1 \mu \mathrm{g}$ of total RNA was used for reverse transcription with M-MLV Reverse Transcriptase (Thermo Fisher Scientific). SYBR Green PCR master mix (Thermo Fisher Scientific) and gene-specific oligonucleotides were used to conduct qPCR analyses. Finally, relative transcription level was calculated using $2^{\Delta \mathrm{Ct}}$ with $A C T 7$ as a control.

RNA-seq analyses. 3'Tag-Seq method was used in this study for RNA-seq analysis $^{53}$. FastQC was used to access raw read quality (www.bioinformatics. babraham.ac.uk/projects/fastqc/). The raw reads were aligned to the Arabidopsis genome using HISAT2 ${ }^{54}$. Data from TAIR10 (www.arabidopsis.org/) was used as the annotation of the Arabidopsis genome. HTseq module was used for counting reads $^{55}$ (htseq.readthedocs.io/en/ master/). Differentially expressed genes in WT and $h y 5$ were identified using the EdgeR ${ }^{56}$. Cutoff and adjusted $P$ value (FDR) for the differential gene expression were setup with $\geq 2$-fold and $\leq 0.05$, respectively. Venn diagrams were generated using the website (http://bioinformatics.psb.ugent. be/webtools/Venn/). Heatmap was generated using Morpheus (https://software. broadinstitute.org/morpheus/). For the heatmap, hierarchical clustering was used with one minus cosine similarity metric combined with average linkage method. Also, GO enrichment analyses were performed using GENE ONTOLOGY (http:// geneontology.org/). GO bar graphs were generated based on the result of the significantly enriched terms with the lowest $P$ value and FDR $(\leq 0.05)$ in GO terms. 
Raw data and processed data for RNA-Seq in Col-0 and hy5-215 can be accessed from the Gene Expression Omnibus database under accession number GSE158939.

Chromatin immunoprecipitation (ChIP) assays. Chromatin Immunoprecipitation (ChIP) assays were conducted as described previously ${ }^{24,46}$. For ChIP assay samples, 6-day-old seedlings of 35S:HY5-GFP in hy5-215 background were transferred to $22^{\circ} \mathrm{C}$ or $28^{\circ} \mathrm{C}$ for $24 \mathrm{~h}$ under continuous white light and then harvested. In brief, samples were crosslinked using $1 \%$ formaldehyde under vacuum for $15 \mathrm{~min}$ and then $2 \mathrm{M}$ glycine was added for additional $5 \mathrm{~min}$ for quenching. Samples were washed with distilled water for five times and ground with mortar and pestle using liquid nitrogen. Ground samples were placed into 1.5 $\mathrm{mL}$ microtube with nuclei isolation buffer $(0.25 \mathrm{M}$ sucrose, $15 \mathrm{mM}$ PIPES, $5 \mathrm{mM}$ $\mathrm{MgCl}_{2}, 60 \mathrm{mM} \mathrm{KCl}, 15 \mathrm{mM} \mathrm{NaCl}, 1 \%$ Triton X-100, $2 \mathrm{mM}$ PMSF, $1 \times$ protease inhibitor cocktail) for $15 \mathrm{~min}$ and then centrifuged at $20,200 \times g$ for $10 \mathrm{~min}$ at $4{ }^{\circ} \mathrm{C}$. Pellets were resuspended with $1 \mathrm{~mL}$ lysis buffer $(50 \mathrm{mM}$ HEPES pH 7.5, $150 \mathrm{mM}$ $\mathrm{NaCl}, 1 \mathrm{mM}$ EDTA, $1 \%$ Triton X-100, 0.1\% SDS, $2 \mathrm{mM}$ PMSF, $1 \times$ protease inhibitor cocktail). Then, sonication of chromatin pellet was performed using digital sonifier (Branson, Model\# PC BD $400 \mathrm{~W} 20 \mathrm{kHz}$ ). ChIP grade anti-GFP (Abcam, ab6556) coupled to dynabeads were used for immunoprecipitation. After washing steps including low salt wash buffer, high salt wash buffer, $\mathrm{LiCl}$ wash buffer, and TE buffer, samples were eluted with elution buffer. By adding $\mathrm{NaCl}$ with final $0.2 \mathrm{M}$, samples were incubated overnight at $65^{\circ} \mathrm{C}$ for reverse crosslinking. Finally, PCR purification kit (QIAGEN) was used for DNA purification after proteinase $\mathrm{K}$ treatment for an hour. Samples without IP were used as input DNA. Enrichment (\% of input) was calculated from each sample relative to their corresponding input.

Microscopy. For light microscopy, seedlings were placed in $22^{\circ} \mathrm{C}$ for 2 days and transferred to $22^{\circ} \mathrm{C}$ or $28^{\circ} \mathrm{C}$ for additional 4 days. Samples were inoculated in $100 \% \mathrm{EtOH}$ overnight and light microscopy was conducted. For fluorescence image, seedlings were placed in $22{ }^{\circ} \mathrm{C}$ for 5 days and transferred to $22^{\circ} \mathrm{C}$ or $28^{\circ} \mathrm{C}$ for additional $4 \mathrm{~h}$ in white light condition. Root images of transgenic seedlings were obtained using Olympus BX53 with $300 \mathrm{~ms}$ exposure time.

Reporting summary. Further information on research design is available in the Nature Research Reporting Summary linked to this article.

\section{Data availability}

RNA sequencing data were deposited into the Gene Expression Omnibus database (accession number GSE158939). Arabidopsis mutants and transgenic lines, as well as plasmids and antibodies generated during the current study are available from the corresponding author upon reasonable request. Source data are provided with this paper.

Received: 18 October 2020; Accepted: 29 May 2021;

Published online: 16 June 2021

\section{References}

1. Lippmann, R., Babben, S., Menger, A., Delker, C. \& Quint, M. Development of wild and cultivated plants under global warming conditions. Curr. Biol. 29, R1326-R1338 (2019)

2. Quint, M. et al. Molecular and genetic control of plant thermomorphogenesis. Nat. Plants 2, 15190 (2016)

3. Casal, J. J. \& Balasubramanian, S. Thermomorphogenesis. Annu. Rev. Plant Biol. 70, 321-346 (2019).

4. Gil, K.-E. \& Park, C.-M. Thermal adaptation and plasticity of the plant circadian clock. New Phytol. 221, 1215-1229 (2019).

5. Legris, M. et al. Phytochrome B integrates light and temperature signals in Arabidopsis. Science 354, 897-900 (2016)

6. Jung, J.-H. et al. Phytochromes function as thermosensors in Arabidopsis. Science 354, 886-889 (2016).

7. Chung, B. Y. W. et al. An RNA thermoswitch regulates daytime growth in Arabidopsis. Nat. Plants 6, 522-532 (2020).

8. Jung, J.-H. et al. A prion-like domain in ELF3 functions as a thermosensor in Arabidopsis. Nature 585, 256-260 (2020).

9. Koini, M. A. et al. High temperature-mediated adaptations in plant architecture require the bHLH transcription factor PIF4. Curr. Biol. 19, 408-413 (2009).

10. Fiorucci, A.-S. et al. PHYTOCHROME INTERACTING FACTOR 7 is important for early responses to elevated temperature in Arabidopsis seedlings. N. Phytologist 226, 50-58 (2020).

11. Stavang, J. A. et al. Hormonal regulation of temperature-induced growth in Arabidopsis. Plant J. 60, 589-601 (2009).

12. Franklin, K. A. et al. PHYTOCHROME-INTERACTING FACTOR 4 (PIF4) regulates auxin biosynthesis at high temperature. Proc. Natl Acad. Sci. USA 108, 20231-20235 (2011)
13. Delker, C. et al. The DET1-COP1-HY5 pathway constitutes a multipurpose signaling module regulating plant photomorphogenesis and thermomorphogenesis. Cell Rep. 9, 1983-1989 (2014).

14. Gangappa, S. N. \& Kumar, S. V. DET1 and HY5 control PIF4-mediated thermosensory elongation growth through distinct mechanisms. Cell Rep. 18, 344-351 (2017).

15. Nieto, C., Lopez-Salmeron, V., Daviere, J. M. \& Prat, S. ELF3-PIF4 interaction regulates plant growth independently of the evening complex. Curr. Biol. 25, 187-193 (2015).

16. Box, MathewS. et al. ELF3 Controls thermoresponsive growth in Arabidopsis. Curr. Biol. 25, 194-199 (2015).

17. Zhu, J. Y., Oh, E., Wang, T. \& Wang, Z. Y. TOC1-PIF4 interaction mediates the circadian gating of thermoresponsive growth in Arabidopsis. Nat. Commun. 7, 13692 (2016)

18. Park, Y.-J. et al. GIGANTEA Shapes the photoperiodic rhythms of thermomorphogenic growth in Arabidopsis. Mol. Plant 13, 459-470 (2020).

19. Ding, L. et al. Two B-Box domain proteins, BBX18 and BBX23, interact with ELF3 and regulate thermomorphogenesis in Arabidopsis. Cell Rep. 25, 1718-1728 e1714 (2018).

20. Lee, H.-J. et al. FCA mediates thermal adaptation of stem growth by attenuating auxin action in Arabidopsis. Nat. Commun. 5, 5473 (2014).

21. Han, X. et al. Arabidopsis transcription factor TCP5 controls plant thermomorphogenesis by positively regulating PIF4 activity. iScience 15 611-622 (2019)

22. Zhou, Y. et al. TCP Transcription factors associate with PHYTOCHROME INTERACTING FACTOR 4 and CRYPTOCHROME 1 to regulate thermomorphogenesis in Arabidopsis thaliana. iScience 15, 600-610 (2019).

23. Balcerowicz, M. PHYTOCHROME INTERACTING FACTORS at the interface of light and temperature signalling. Physiol. Plant. 169, 347-356 (2020).

24. Lee, S., Paik, I. \& Huq, E. SPAs promote thermomorphogenesis by regulating the phyB-PIF4 module in Arabidopsis. Development 147, $\operatorname{dev} 189233(2020)$

25. Lee, S., Zhu, L. \& Huq, E. An autoregulatory negative feedback loop controls thermomorphogenesis in Arabidopsis. PLoS Genet. 17, e1009595 (2021).

26. Strock, C. F. et al. Seedling root architecture and its relationship with seed yield across diverse environments in Phaseolus vulgaris. Field Crops Res. 237, 53-64 (2019)

27. McMichael, B. L. \& Quisenberry, J. E. The impact of the soil environment on the growth of root systems. Environ. Exp. Bot. 33, 53-61 (1993).

28. El Hassouni, K. et al. Root system architecture and its association with yield under different water regimes in durum wheat. Crop Sci. 58, 2331-2346 (2018).

29. Veerappa, R. et al. Ectopic expression of a pea apyrase enhances root system architecture and drought survival in Arabidopsis and soybean. Plant, Cell Environ. 42, 337-353 (2019).

30. Martins, S. et al. Brassinosteroid signaling-dependent root responses to prolonged elevated ambient temperature. Nat. Commun. 8, 309 (2017).

31. Hanzawa, T. et al. Cellular auxin homeostasis under high temperature is regulated through a SORTING NEXIN1-dependent endosomal trafficking Pathway. Plant Cell 25, 3424-3433 (2013).

32. Feraru, E. et al. PILS6 is a temperature-sensitive regulator of nuclear auxin input and organ growth in Arabidopsis thaliana. Proc. Natl Acad. Sci. USA 116, 3893-3898 (2019).

33. Wang, R. et al. HSP90 regulates temperature-dependent seedling growth in Arabidopsis by stabilizing the auxin co-receptor F-box protein TIR1. Nat. Commun. 7, 10269 (2016).

34. Gaillochet, C. et al. HY5 and phytochrome activity modulate shoot-to-root coordination during thermomorphogenesis in Arabidopsis. Development 147, dev192625 (2020).

35. Oyama, T., Shimura, Y. \& Okada, K. The Arabidopsis HY5 gene encodes a bZIP protein that regulates stimulus-induced development of root and hypocotyl. Genes Dev. 11, 2983-2995 (1997).

36. Toledo-Ortiz, G. et al. The HY5-PIF regulatory module coordinates light and temperature control of photosynthetic gene transcription. PLoS Genet. 10, e1004416 (2014).

37. Burko, Y. et al. Chimeric activators and repressors define HY5 activity and reveal a light-regulated feedback mechanism. Plant Cell 32, 967-983 (2020).

38. Qiu, T. et al. The tissue-specific and developmentally regulated expression patterns of the SAUR41 subfamily of SMALL AUXIN UP RNA genes. Plant Signal. Behav. 8, e25283 (2013).

39. Li, Z.-G. et al. Three SAUR proteins SAUR76, SAUR77 and SAUR78 promote plant growth in Arabidopsis. Sci. Rep. 5, 12477 (2015).

40. Ren, H. \& Gray, WilliamM. SAUR Proteins as effectors of hormonal and environmental signals in plant growth. Mol. Plant 8, 1153-1164 (2015).

41. Sun, J., Qi, L., Li, Y., Zhai, Q. \& Li, C. PIF4 and PIF5 transcription factors link blue light and auxin to regulate the phototropic response in Arabidopsis. Plant Cell 25, 2102-2114 (2013). 
42. Lee, J. et al. Analysis of transcription factor HY5 genomic binding sites revealed its hierarchical role in light regulation of development. Plant Cell 19, 731-749 (2007).

43. Zhang, H. Y. et al. Genome-wide mapping of the HY5-mediated gene networks in Arabidopsis that involve both transcriptional and posttranscriptional regulation. Plant J. 65, 346-358 (2011).

44. Wang, W. et al. Direct phosphorylation of HY5 by SPA kinases to regulate photomorphogenesis in Arabidopsis. New Phytol. 230, 2311-2326 (2021).

45. Hardtke, C. S. et al. HY5 stability and activity in arabidopsis is regulated by phosphorylation in its COP1 binding domain. EMBO J. 19, 4997-5006 (2000).

46. Paik, I. et al. A phyB-PIF1-SPA1 kinase regulatory complex promotes photomorphogenesis in Arabidopsis. Nat. Commun. 10, 4216 (2019).

47. Challinor, A. J. et al. A meta-analysis of crop yield under climate change and adaptation. Nat. Clim. Change 4, 287-291 (2014).

48. Uga, Y. et al. Control of root system architecture by DEEPER ROOTING 1 increases rice yield under drought conditions. Nat. Genet. 45, 1097-1102 (2013).

49. Burko, Y., Gaillochet, C., Seluzicki, A., Chory, J. \& Busch, W. Local HY5 activity mediates hypocotyl growth and shoot-to-root communication. Plant Commun. 1, 100078 (2020).

50. Chen, X. et al. Shoot-to-root mobile transcription factor HY5 coordinates plant carbon and nitrogen acquisition. Curr. Biol. 26, 640-646 (2016).

51. Osterlund, M. T., Hardtke, C. S., Wei, N. \& Deng, X. W. Targeted destabilization of HY5 during light-regulated development of Arabidopsis. Nature 405, 462-466 (2000).

52. Chen, D. et al. Antagonistic basic helix-loop-helix/bZIP transcription factors form transcriptional modules that integrate light and reactive oxygen species signaling in Arabidopsis. Plant Cell 25, 1657-1673 (2013).

53. Lohman, B. K., Weber, J. N. \& Bolnick, D. I. Evaluation of TagSeq, a reliable low-cost alternative for RNAseq. Mol. Ecol. Resour. 16, 1315-1321 (2016).

54. Kim, D., Langmead, B. \& Salzberg, S. L. HISAT: a fast spliced aligner with low memory requirements. Nat. Methods 12, 357-360 (2015).

55. Anders, S., Pyl, P. T. \& Huber, W. HTSeq-a Python framework to work with high-throughput sequencing data. Bioinformatics 31, 166-169 (2015).

56. Robinson, M. D., McCarthy, D. J. \& Smyth, G. K. edgeR: a bioconductor package for differential expression analysis of digital gene expression data. Bioinformatics 26, 139-140 (2010).

\section{Acknowledgements}

We thank members of the Huq laboratory for critical reading of the manuscript. We thank Drs. Mijeong Kim and Taeyoung Lee for comments analyzing RNA-seq. This work was supported by grants from the National Science Foundation (MCB-2014408) and National Institute of Health (NIH) (GM-114297) to E.H., and Integrative Biology (IB)
Research Fellowship grant from the University of Texas at Austin to S.L. The authors acknowledge the Texas Advanced Computing Center (TACC) at The University of Texas at Austin for providing High Performance Computing, visualization, and database resources that have contributed to the research results reported in this paper.

\section{Author contributions}

S.L., W.W., and E.H. conceived the study and designed the experiments. S.L. and W.W. carried out the experiments. S.L., W.W., and E.H. analyzed the data. S.L. and E.H. wrote the paper.

\section{Competing interests}

The authors declare no competing interests.

\section{Additional information}

Supplementary information The online version contains supplementary material available at https://doi.org/10.1038/s41467-021-24018-7.

Correspondence and requests for materials should be addressed to E.H.

Peer review information Nature Communications thanks Jigang Li and the other, anonymous reviewer(s) for their contribution to the peer review of this work. peer review reports are available.

Reprints and permission information is available at http://www.nature.com/reprints

Publisher's note Springer Nature remains neutral with regard to jurisdictional claims in published maps and institutional affiliations.

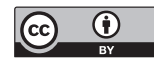

Open Access This article is licensed under a Creative Commons Attribution 4.0 International License, which permits use, sharing, adaptation, distribution and reproduction in any medium or format, as long as you give appropriate credit to the original author(s) and the source, provide a link to the Creative Commons license, and indicate if changes were made. The images or other third party material in this article are included in the article's Creative Commons license, unless indicated otherwise in a credit line to the material. If material is not included in the article's Creative Commons license and your intended use is not permitted by statutory regulation or exceeds the permitted use, you will need to obtain permission directly from the copyright holder. To view a copy of this license, visit http://creativecommons.org/ licenses/by/4.0/.

(C) The Author(s) 2021 